\title{
SAGIMA: An Easy-to-Use and Low Cost WEB-PACS System for an Optimal Access and Management of a Digital Angiography Database
}

\author{
P Lombillo-Biosca ${ }^{1}$, D Moratal ${ }^{1}$, DR García-Sánchez ${ }^{1}$, V Bodí-Peris ${ }^{2}$, \\ C Gómez ${ }^{2}$, C Sánchez-Meléndez ${ }^{3}$, JJ Rieta $^{1}$ \\ ${ }^{1}$ Departamento de Ingeniería Electrónica, Universidad Politécnica de Valencia, Spain \\ ${ }^{2}$ Departamento de Cardiología, Hospital Clínico Universitario de Valencia, Spain \\ ${ }^{3}$ DIEEA, EUP Cuenca, Universidad de Castilla-La Mancha, Spain
}

\begin{abstract}
Over several years, digital angiography studies from the Hemodynamic Unit of the Hospital Clínico Universitario (Valencia, Spain) have been stored in CD's using first revisions of DICOM 3.0. In order to centralize the management and facilitate the access to these studies and reports, an easy to use and low cost WEB-PACS system that we have called SAGIMA has been developed in close collaboration between the BET Research Group of the Universitat Politècnica de València and the Cardiology Department of the Hospital Clínico Universitario de Valencia.
\end{abstract}

\section{Introduction}

Nowadays PACS (Picture Archive and Communication System) have become the facto standard to store and access the results obtained from imaging study types like MRI, Digital Angiography, X-Ray, CT and so, but in some cases it is not possible to install a PACS in a hospital, sometimes because of its cost and in other cases because the structure of the hospital does not allow its installation itself. Although those inconvenient, most of the hospitals store studies over digital supports like CD-ROM, and its manual managing generates several problems of storage, slow access, bad identification and in the worst cases studies are damaged or lost.

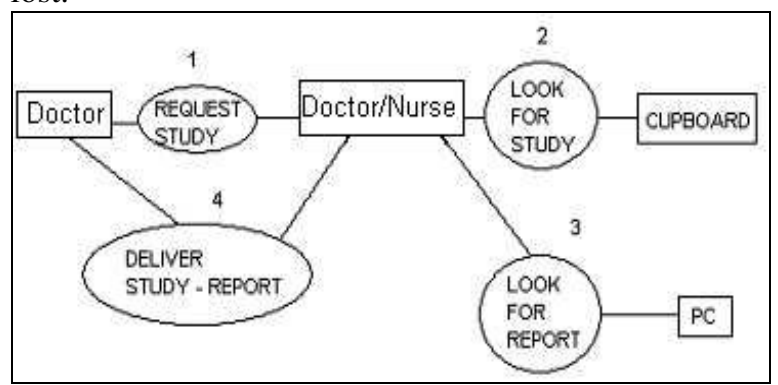

Figure 1. Sequence model of the problem

\section{Objectives}

In order to mitigate those problems, the BET Group (Bioingeniería, Electrónica y Telemedecina) of the Universidad Politécnica de Valencia has developed adhoc in collaboration with the Hemodinamic Unit of the Hospital Clínico Universitario de Valencia, a low cost system called SAGIMA, which allows storing in a database all the studies, reports, and user accounts, while accessing to the studies and reports is allowed through internet.

The main objectives of this project were: making a reengineering of the process of storing, managing and accessing the studies to reduce the delay that exists among the request and delivery of a study and to allow remote concurrent study reviews. Furthermore with this project we wanted to centralize all the information, reducing the possibilities of study damaging and what is more important, getting a digital patient history record which helps dramatically during diagnostic.

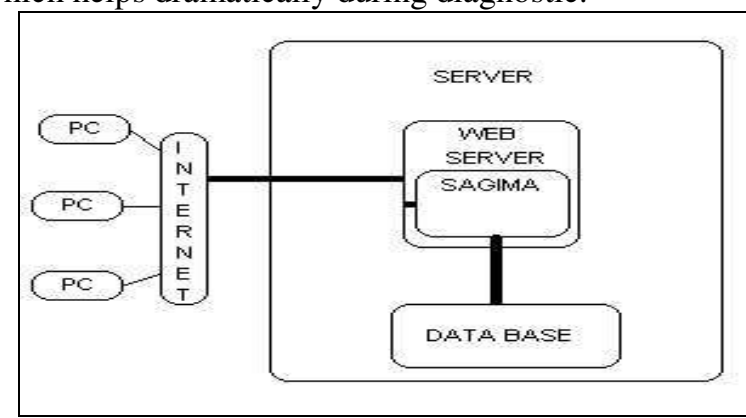

Figure 2. The scheme of the architecture of our proposed solution.

\section{Materials and methods}

\section{Architecture}

The system has been developed with a 3 tier architecture, where the Client / Server / Data, have been 
clearly separated in order to decouple the design.

The Client is supported by the user's Navigator and the WEB pages, while the Server side is implemented by the Tomcat application container Server (can be used as WEB Server) and our application SAGIMA, and the Data is supported over a MySQL database manager.

\section{Hardware}

The Tomcat Server, MySQL, SAGIMA, and all the data from studies and reports, have been installed in a Massive Storage Server which was configured ad-hoc.

The Server has a 3.0 Pentium IV processor with 1 Gbyte of RAM memory and 800 Gbyte of hard disk, all managed by Windows 2000 operative system.

The configuration allows storage of around 3 years of study records, and data transfers of around 1 Mbyte of speed which allows accessing to the studies in just a few 3-10 seconds depending on the study size.

\section{The Software}

The software used to develop SAGIMA is available for free, with an important amount of money saving.

The tools for analysis and design were ArgoUML wich permits the creation of UML diagrams and DBDesigner 4 which was used to model the Data Base logic. The implementation of the code was done using the RAD (Rapid Application Development) tool NetBeans 3.6 for JAVA programming which is the language selected because of its good properties: robust, highly web oriented, object oriented, and with a very good support.

To help us the supporting of DICOM 3.0 functions, we used DCM4CHE Java API and PIXELMED.

\section{The Methodology}

The methodology used to develop SAGIMA was guided by RUP (Rational Unified Process) Software Engineering methodology, but close to the Agile methods, to make the process more dynamic, and provide as fast as possible the software to start using it. The documents were generated using UML standard.

\section{Elaboration phase}

First, several meetings were made with the client medics and nurses, in order to consider all the information, specify all the requirements needed and highlight important concepts that permit to model the data and situation.

\section{The Analysis phase}

In this phase we worked over the information obtained from Elaboration Phase, to find which were the actions that users should do with the System, that is the Use Cases.

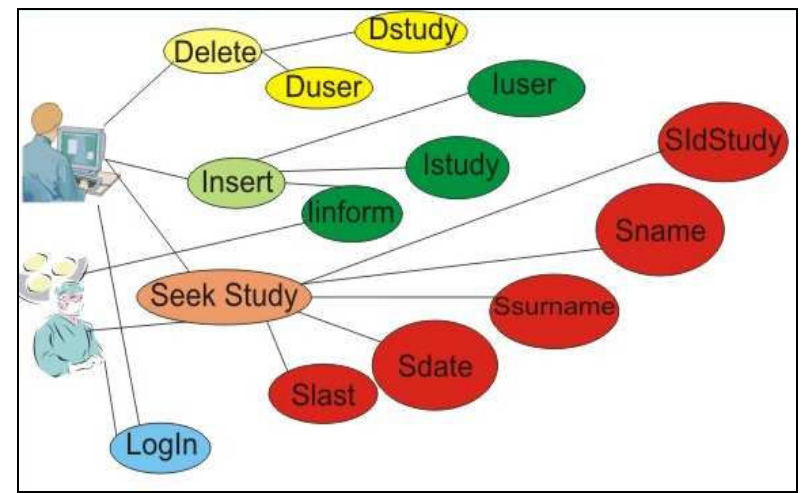

Figure 3. Use Case diagram

Each use case specifies a full action over the system to get a result.

Use cases helped us to conform the Conceptual model, in order to emphasize the concepts that were really important for us like Study, Series, Images, Patient, etc.

\section{The design phase}

With the Use case and Conceptual diagrams we started working on the logic that had to support those use cases, generating Sequential Diagrams for each use case and later in more depth Collaboration Diagrams which helped to realize which were the object we should create in the Implementation phase.

In order to complete this stage, we used several software patterns like the MVC (Model View Controller) pattern, which greatly fits for the purpose we wanted, and Façade pattern that permits the decoupling at the component level of SAGIMA and the data base used.

\section{The implementation phase}

Finally we used all the information acquired in previous phases to implement the solution in real code.

For SAGIMA the technology used was server side Servlets, JSP (Java Script Pages) and Beans as recommended by SUN to implement the MVC pattern. This design helps to make the system more modular and easy to maintain.

Furthermore, several tweaks were implemented to improve the whole operation, those are Connection Pooling, which improves dramatically the access to the DataBase, the application SAGIMA was decoupled from the database so it can be used other databases, and several parameters were taken out to configuration files so they can be modified to be reconfigured in case new conditions should be achieved, like the maximum number of clients allowed at once, several passwords and so.

The program SAGIMA-CD was developed using SWING, which greatly helped to design its interface which was mainly designed as medics and nurses decided, so they do not have to spend so much time 
learning how to use it.

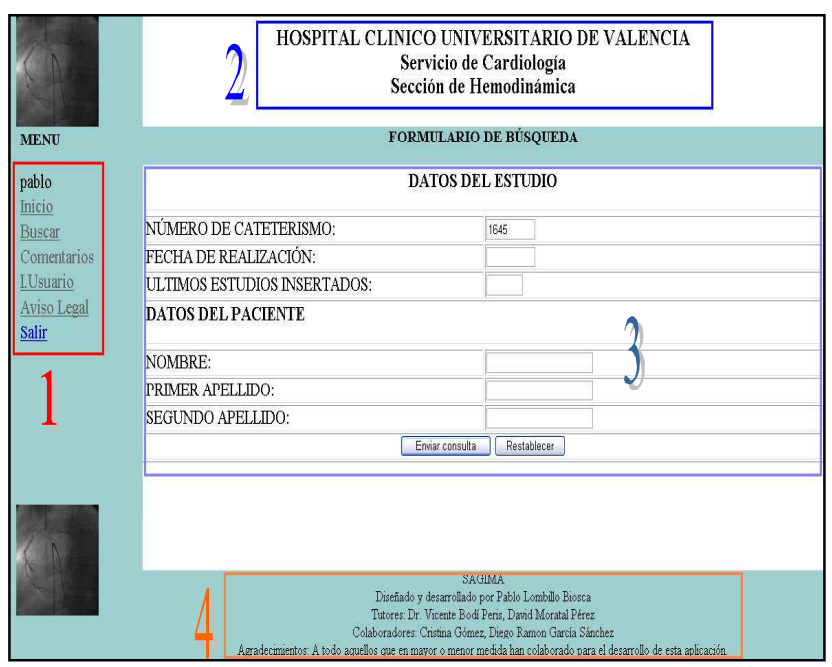

Figure 4. Detail of SAGIMA's searching web page. 1 Menu, 2 Title, 3 Form, 4 Acknowledgements

\section{Results}

The result of this project is a $24 \times 7 \times 365$ available system, which through SAGIMA-CD allows storing and managing all the studies, reports and user accounts, while using SAGIMA web, users can access the studies from their computer with just a few clicks to download them, read the report or attach a comment to a study.

As stated by LOPD (Ley Orgánica de Protección de Datos) law all the data is transmitted via SSL (Secure Shell Layer) including user's login and password in order to protect patient's privacy. Not only the client gets the data in a safer channel but we ensure that the data is not modified by anyone since it is sent till it is received.

\subsection{SAGIMA WEB}

Through the web of SAGIMA users can do several actions. Normal Users must login and then may seek studies, reports or just attach comments to the studies, just filling simple forms that allow to search by several keys. Administrators can create or erase new users, and are allowed to do normal Users actions too.

Although all users can enter all pages, actions can not be completed unless the user has privileges to do it, so this way we introduce a second level of security after the login and encryption.

The interface is a very basic one, keeping in mind that it should be simple in order to be easy to use, and light designed to prevent distractions. However visual design was not the objective of this project and it fits very well with the needs of the unit.

While the main page remains the same, the body page is generated dynamically, containing several links according to the searching type or action result, so the user can navigate easily till he gets what he wants.

\begin{tabular}{|c|c|c|c|c|}
\hline N.CATETERISMO & REALIZACIÓN & J INFORMES & NOMBRE & $\begin{array}{l}\text { FECHA } \\
\text { NACIMIENTO }\end{array}$ \\
\hline 3 & 2004-04-02 & Informe & $\begin{array}{l}\text { PABLO } \\
\text { LOMBILLO } \\
\text { BIOSCA }\end{array}$ & 1944-10-09 \\
\hline 2 & 2004-04-02 & Informe & \begin{tabular}{|l} 
DAVID \\
MORATAL \\
PEREZ
\end{tabular} & 1944-10-09 \\
\hline 1 & 2004-04-02 & $\begin{array}{l}\text { Informe, }, \text { Informe } \\
\text { Angioplastia } \\
\end{array}$ & $\begin{array}{l}\text { VICENT BODI } \\
\text { PERIS } \\
\end{array}$ & 1944-10-09 \\
\hline
\end{tabular}

Figure 5. Result after seeking last 3 studies inserted. Links to 1 . Studies, 2. Reports, 3. Single patient's study list

In Figure 6, we can see the final page that allows downloading each DICOM3.0 file of the study. It is generated with several links, one to the rest of the studies from the same patient, one or two links to the reports, and one link to each file in the study, with a thumbnail that corresponds to the middle frame of the video.

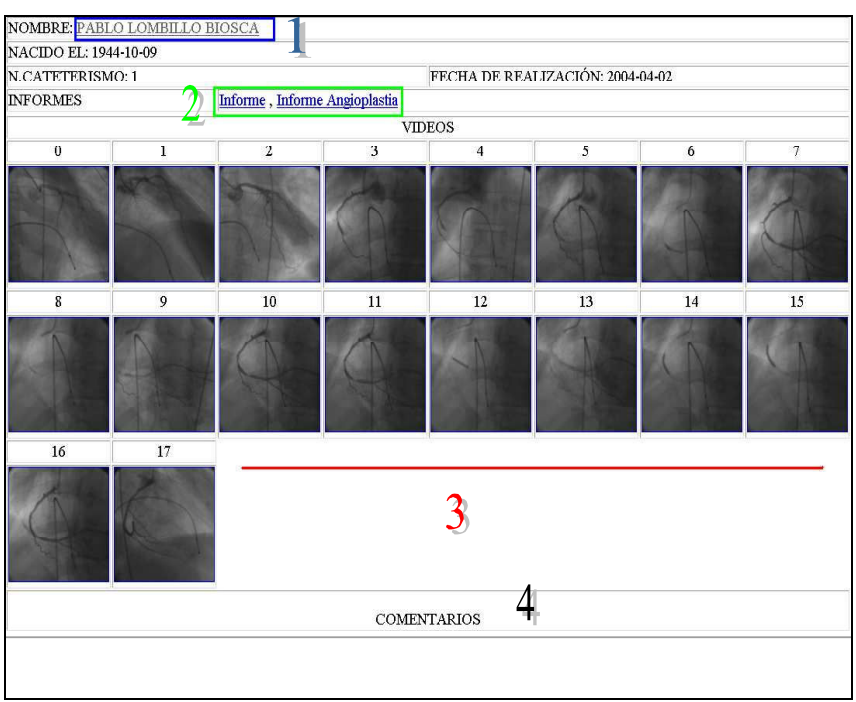

Figure 6. Example of a the result of the seek for study ID 1. Link to patient studies 2. Link to reports 3. Links with thumbnails of the videos to allow a preview 4. Comments attached to the study.

\subsection{SAGIMA-CD}

SAGIMA-CD is a stand-alone program which runs inside the system server. That way of implementing was due to the size of DICOM3.0 study files, which were around 20 files per study and 5 Mbytes per file. That study size would had collapsed the Unit's network while updating the database.

Its interface was developed to fit users desires so they could use it without training. 
Administrators can use it to update the database of SAGIMA with new studies just filling a few fields, and the program loads in short time (15-50 seconds depending on the study size) all the DICOM3.0 files and generates the thumbnails automatically.

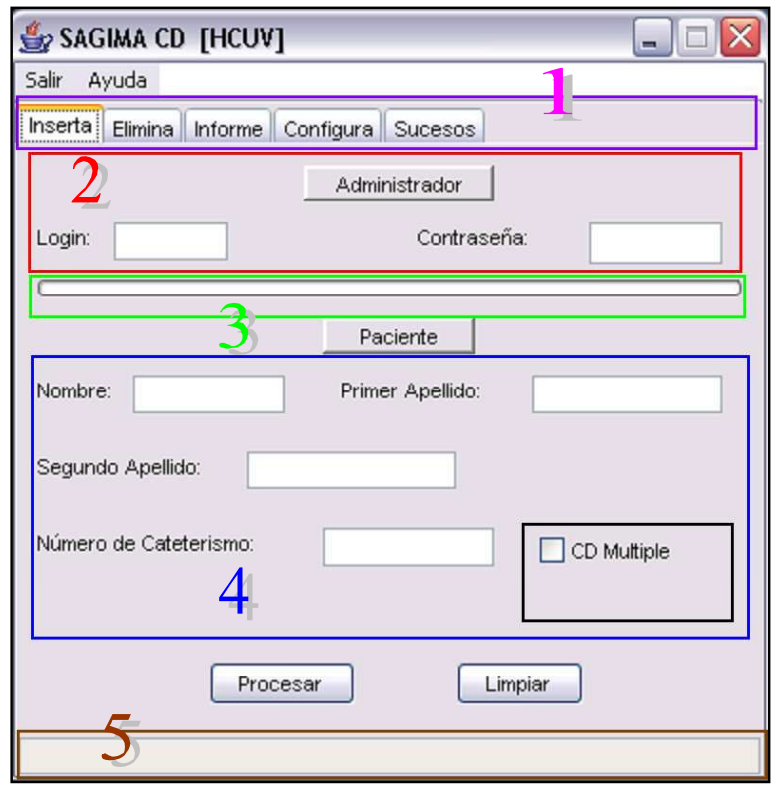

Figure 7. Main interface of SAGIMA-CD. 1 Window handler 2. Administrator Login form 3. Progress bar 4. Study/Patient form 4. Event message bar.

After inserting the new study, they can attach automatically the report.

Another property of SAGIMA-CD is that it is possible to attach multiple reports at the same time, so the most optimal way of working with it is to insert each study when is finished, and wait till the last is inserted, to insert all the reports with just one click.

Studies can be erased from the system with just 2 clicks and they can erase the study completely or keep the report.

SAGIMA-CD has a panel where Administrator can configure parameters like source file directory, target directory, report source to ensure that it can work in every system.

Finally we want to remember that the programming language used, JAVA, allows to use the system in every computer that has installed a Java Virtual Machine.

\section{Conclusion}

After finishing this project, we can confirm that main objectives have been achieved for the needs of the Unit:

- The process of requesting a study or report does not last hours or days, now in a few seconds the doctor can access the information from his/her computer (much more, from every computer of the hospital).

- Several specialists can review the same study at one time, and this framework helps on moving to a multidisciplinary diagnosis work cycle.

- All the data is centralized, no one manipulates original copies. This way we protect the studies from damage or loss.

- Studies and reports are linked, so they are no more dissociated which results in the conformation of a digitalized clinical patient record, helping in following diagnosis.

- With primary objectives achieved we realized about several changes in the efficiency of the unit:

- Time saved in accessing studies and reviewing them can be used to complete other important activities.

- The work cycle has become smoother. Now the amount of study review work per day has become more balanced.

- We have registered an increase on the number of study and report reviews per day. While no more than 3 or 4 reviews per day could be done with manual management, now around 40 reviews per day are done, actually it could be more.

We have realized that a small tool like we have designed can be interesting for those small hospital units that can not buy a PACS because of the prize or old hospital building.

\section{Acknowledgements}

This project has been partially subsidized by the action IIARC0/2004/249 of the Conselleria d'Empresa Universitat i Ciéncia de la Generalitat Valenciana.

\section{References}

[1] ACR/NEMA. The DICOM 3.0 standard, 2003

[2] Philips Medical Systems. DICOM Cookbook

[3] Singh I, Stearns B, Johnson M and the Enterprise Team. Designing Enterprise Applications. AddisonWesley, 2002

[4] Conallen J. Building WEB applications with UML. Addison-Wesley, 2002

[5] Hall M. Core Servlets and Java Server Pages. Prentice Hall, 2002

[6] Ford N. Art of Java WEB Development. Manning Publications, 2004

[7] Dubois P. MySQL Cookbook. O'Reilly, 2004

[8] Brittain J, Darwin IF. Tomcat: the definitive guide. O'Reilly, 2003

[9] University Hospital of Geneva: Medical Informatics Department, Numerical Engineering Unit. OSIRIS User Manual V 3.0; UIN/HCUG: 1995-1996 